\title{
COVID-19: Getting to the heart of the issue
}

\author{
Beth Woodward ${ }^{1}$ and Muhammed Kumali ${ }^{2}$ \\ ${ }^{1}$ University of Birmingham \\ ${ }^{2}$ University of London St George's
}

May 14, 2020

\section{COVID-19: The heart of the issue}

Beth Woodward BMedSc (Hons) ${ }^{1}$, Muhammed Kermali ${ }^{2}$

1. College of Medical and Dental Sciences, University of Birmingham, Birmingham, UK

2. St. George's, University of London, London, UK

Corresponding author:

Beth Woodward

BMedSc (Hons)

College of Medical and Dental Sciences

University of Birmingham

Birmingham, UK

e-mail: blw472@student.bham.ac.uk

Tel: 07947766140

Funding: none obtained

Conflict of Interest: none to be declared

Key words: COVID-19, angiotensin, ACEi

BW and MK contributed equally.

\section{To the Editor:}

We read with great interest the review by Khan et al. who discussed the emerging role of cardiovascular disease and the progression of the novel Coronavirus disease 19 (COVID-19). ${ }^{1}$ As part of their review, the authors discussed a broad range of cardiovascular effects being observed during and post infection in this disease. We commend their conclusions that appropriate triage and risk stratification is essential in patients with cardiovascular disease (CVD) and COVID-19. We encourage the need for further evidence regarding the mechanisms of CVD and particularly the use of therapeutics. This is of particular importance given the global concern about the use of renin-angiotensin-aldosterone system (RAAS) inhibitors in the mechanisms of severe acute respiratory distress syndrome coronavirus 2 (SARS-CoV-2).

SARS-CoV-2 utilises the Angiotensin converting enzyme 2 (ACE2) cell membrane protein on lung alveolar epithelial cells to enter host cells. ${ }^{1}$ This ACE2 enzyme is key in the cascade of the (RAAS) so the authors highlight of the importance understanding this further was justified. RAAS inhibitors contributing to increased severity of COVID-19, most commonly in hypertension, is a logical hypothesis due to their mode 
of action. However, it is currently unclear whether this virus's interaction with ACE2 causes dysregulation or interference of downstream effectors. Early observational studies concluded that use of RAAS inhibitors may not be associated with SARS-CoV-2 infection, or more severe Covid-19 progression. One retrospective multi-centre study with 1128 participants (188 on a RAAS inhibitor) suggested that in hospitalised COVID19 patients with pre-existing hypertension, RAAS inhibitors had better outcomes compared with non-RAAS inhibitoruse. $^{2}$ This supports continuing their use if already prescribed but recognises the significant risk for confounding variables in their methodology. ${ }^{2}$

Discontinuing these medications may potentially put patients at greater risk of complications, directly due to SARS-CoV-2 infection or indirectly from a period of blood pressure instability and attenuating their known cardio- and renal-protective effects. ${ }^{3}$ Furthermore, the authors highlighted that cardiovascular complications and myocardial injury have also been observed peri- and post-infection with SARS-CoV-2, even in patients without pre-existing $\mathrm{CVD}^{1}$. Given these mechanisms are yet to be elucidated and likely to be multifactorial, this further supports this notion.

We support the conclusion made that there is currently limited evidence to recommend their use should be discontinued prophylactically and in confirmed cases. ${ }^{3,4}$ It is pivotal further pre-clinical studies are carried out to understand the cardiovascular mechanisms in COVID-19. We emphasise the need to elucidate mechanisms of RAAS inhibitors in SARS-CoV-2 infection. Robust and collaborative human studies are needed to apply this understanding in a meaningful way, where major confounding variables, such as advancing age, can be accounted for.

References

1. Khan, I.H., Zahra, S.A., Zaim, S., Harky, A., 2020. At the heart of COVID-19. J Card Surg. 1-2.

2. Zhang, P., Zhu, L., Cai, J., Lei, F., Qin, J.-J., Xie, J., Liu, Y.-M., Zhao, Y.-C., Huang, Xuewei, Lin, L., Xia, M., Chen, M.-M., Cheng, X., Zhang, X., Guo, D., Peng, Y., Ji, Y.-X., Chen, J., She, Z.-G., Wang, Y., Xu, Q., Tan, R., Wang, H., Lin, J., Luo, P., Fu, S., Cai, H., Ye, P., Xiao, B., Mao, W., Liu, L., Yan, Y., Liu, M., Chen, M., Zhang, X.-J., Wang, X., Touyz, R.M., Xia, J., Zhang, B.-H., Huang, Xiaodong, Yuan, Y., Rohit, L., Liu, P.P., Li, H., 2020. Association of Inpatient Use of Angiotensin Converting Enzyme Inhibitors and Angiotensin II Receptor Blockers with Mortality Among Patients With Hypertension Hospitalized With COVID-19. Circulation Research.

3. Jarcho, J.A., Ingelfinger, J.R., Hamel, M.B., D'Agostino, R.B., Harrington, D.P., 2020. Inhibitors of the Renin-Angiotensin-Aldosterone System and Covid-19. N Engl J Med NEJMe2012924.

4. Khashkhusha, T.R., Chan, J.S.K., Harky, A., 2020. ACE inhibitors and COVID-19: We don't know yet. J Card Surg. 